\section{(6) OPEN ACCESS}

\title{
AMPA/kainate glutamate receptors contribute to inflammation, degeneration and pain related behaviour in inflammatory stages of arthritis
}

\author{
Cleo S Bonnet, ${ }^{1}$ Anwen S Williams, ${ }^{2}$ Sophie J Gilbert, ${ }^{1}$ Ann K Harvey, ${ }^{3}$ \\ Bronwen A Evans, ${ }^{4}$ Deborah J Mason ${ }^{5}$
}

Handling editor Tore K Kvien

- Additional material is published online only. To view please visit the journal online (http://dx.doi.org/10.1136/ annrheumdis-2013-203670).

${ }^{1}$ Arthritis Research UK Biomechanics and Bioengineering Centre, School of Biosciences, Cardiff University, Cardiff, UK ${ }^{2}$ Institute of Infection and Immunity, School of Medicine, Cardiff University, Cardiff, UK ${ }^{3}$ Arthritis Research UK Biomechanics and Bioengineering Centre, School of Medicine, Cardiff University, Cardiff, UK ${ }^{4}$ Institute of Molecular and Experimental Medicine, School of Medicine, Cardiff University, Cardiff, UK

${ }^{5}$ Division of Pathophysiology and Repair, School of Biosciences, Cardiff University, Cardiff, UK

\section{Correspondence to}

Dr Deborah Mason, Division of Pathophysiology and Repair, Arthritis Research UK Biomechanics and Bioengineering Centre, School of Biosciences, Cardiff University, Museum Avenue, Cardiff CF10 3AX, UK; MasonDJ@cardiff.ac.uk

Received 22 March 2013 Revised 12 August 2013 Accepted 20 September 2013 Published Online First 14 October 2013

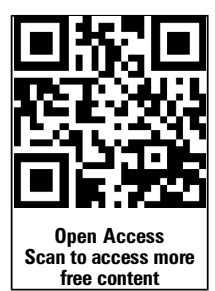

CrossMark

To cite: Bonnet $C S$, Williams AS, Gilbert SJ, et al. Ann Rheum Dis 2015;74:242-251.

\section{ABSTRACT}

Objectives Synovial fluid glutamate concentrations increase in arthritis. Activation of kainate (KA) and $\alpha$ amino-3-hydroxy-5-methyl-4-isoxazolepropionic acid (AMPA) glutamate receptors (GluRs) increase interleukin6 (IL-6) release and cause arthritic pain, respectively. We hypothesised that AMPA and KA GluRs are expressed in human arthritis, and that intra-articular NBQX (AMPA) KA GluR antagonist) prevents pain and pathology in antigen-induced arthritis (AIA).

Methods GluR immunohistochemistry was related to synovial inflammation and degradation in osteoarthritis $(\mathrm{OA})$ and rheumatoid arthritis (RA). A single intraarticular NBQX injection was given at induction, and knee swelling and gait of AIA and AIA+NBQX rats compared over 21 days, before imaging, RT-qPCR, histology and immunohistochemistry of joints. Effects of NBQX on human primary osteoblast (HOB) activity were determined.

Results AMPAR2 and KA1 immunolocalised to remodelling bone, cartilage and synovial cells in human $\mathrm{OA}$ and RA, and rat AIA. All arthritic tissues showed degradation and synovial inflammation. NBQX reduced GluR abundance, knee swelling ( $p<0.001$, days 1-21), gait abnormalities (days 1-2), end-stage joint destruction $(p<0.001)$, synovial inflammation $(p<0.001)$, and messenger RNA expression of meniscal IL- $6(p<0.05)$ and whole joint cathepsin $K(p<0.01)$. X-ray and MRI revealed fewer cartilage and bone erosions, and less inflammation after NBQX treatment. NBQX reduced $\mathrm{HOB}$ number and prevented mineralisation.

Conclusions AMPA/KA GluRs are expressed in human $O A$ and $R A$, and in $A I A$, where a single intra-articular injection of NBQX reduced swelling by $33 \%$, and inflammation and degeneration scores by $34 \%$ and $27 \%$, respectively, exceeding the efficacy of approved drugs in the same model. AMPA/KA GluR antagonists represent a potential treatment for arthritis.

\section{INTRODUCTION}

Inflammation underlies pathology in osteoarthritis $(\mathrm{OA})^{1-3}$ and rheumatoid arthritis (RA). ${ }^{4}$ Nonsteroidal anti-inflammatory drugs, corticosteroids and anti-cytokine treatments that have revolutionised RA treatment ${ }^{4}$ also relieve OA symptoms with varying success. $^{5-9}$ Here, we investigate whether glutamate receptor (GluR) antagonists represent a new treatment targeting inflammatory stages of arthritis.
Synovial fluid (SF) glutamate concentrations increase 52-fold in RA (326 $\mu \mathrm{M})$ and 42-fold in OA $(266 \mu \mathrm{M})^{10}$ and in arthritis animal models. ${ }^{11}{ }^{12} \mathrm{In}$ RA, high SF glutamate correlates with increased inflammatory mediators. ${ }^{13}{ }^{14}$ Glutamate is now known to signal in various 'non-excitable' cells, ${ }^{15-17}$ being released by nerves, macrophages, lymphocytes, synoviocytes, osteoblasts, osteoclasts and chondrocytes, ${ }^{11} 141819$ and acting on ionotropic glutamate receptors (iGluRs) and metabotropic GluRs in multiple joint cell types. ${ }^{18} 2021$ GluRs regulate peripheral pain, ${ }^{22}$ cytokine and matrix metalloproteinase (MMP) release $^{20}$ synoviocyte proliferation $^{23} 24$ and immune responses. ${ }^{21}$ Therefore, GluR antagonists represent potential drugs with multimodal activity against arthritis symptoms.

Intra-articular injections of iGluR antagonists have been shown to inhibit pain for $24 \mathrm{~h}$ in murine carrageenan-induced arthritis (MK801, NBQX), ${ }^{25}$ suppress inflammatory pain for $24 \mathrm{~h}$ in arthritic mice $\left(\right.$ GYKI 52466, 1-NAS) ${ }^{26}$ and alleviate allodynia over 7 days in complete Freund's adjuvant (CFA)-induced arthritis when combined with a substance P receptor antagonist and dexamethasone. ${ }^{27}$ Of two studies investigating the effects of GluR antagonists on arthritic pathology, one showed that a single intra-articular treatment targeting all iGluRs did not affect cartilage erosion in CFA arthritis, ${ }^{27}$ and the other revealed that continual systemic administration of memantine (N-methyl-D-aspartate receptor (NMDAR) antagonist) alleviated synovitis and joint destruction in collagen-induced arthritis (CIA). ${ }^{21}$ Long-term effects of single treatments of GluR antagonists on arthritic pain, inflammation and pathology are unknown, and no studies have investigated the pathological effects of $\alpha$-amino-3-hydroxy-5methyl-4-isoxazolepropionic acid (AMPA)/kainate (KA) GluR antagonists.

Since SF glutamate concentrations increase immediately after arthritis induction, ${ }^{11}$ AMPA GluRs mediate arthritic pain ${ }^{26}$ and KA GluR activation causes interleukin-6 (IL-6) release, ${ }^{20}$ a critical mediator of arthritic joint destruction, ${ }^{28}$ we hypothesised that early intra-articular intervention with NBQX (AMPA/KA GluR antagonist) would reduce pain, inflammation and pathology in inflammatory arthritis. The antigen-induced arthritis (AIA) rat model was used as it shares pathological features of human inflammatory arthritis, ${ }^{29}$ has defined IL- 6 release profiles ${ }^{29}$ and does not require 
analgesia. NBQX was administered at arthritis induction, prior to peak IL-6 concentrations, and gait, knee swelling, synovial inflammation, GluR expression and joint degeneration determined. To assess relevance to inflammatory processes in human arthritis, we investigated AMPA and KA GluR expression in human RA and OA knees.

\section{METHODS}

\section{Patients}

Matched synovium and medial tibial plateaux (MTP) were obtained (informed consent, Ref: 10/MRE0928) from randomly selected patients undergoing total knee replacement for OA (two women, one man) or RA (one man).

\section{Animals}

Procedures were performed according to Home Office guidelines on male Lewis rats ( $200 \mathrm{~g}$, Harlan, UK) housed in groups of three (12 h light-dark cycles, ad libitum food and water).

\section{Arthritis and treatments}

After two subcutaneous injections of methylated bovine serum albumin (mBSA; $0.5 \mathrm{mg} / \mathrm{mL}$; Sigma) emulsified with an equal volume of CFA (0.25 mg Mycobacterium tuberculosis, Sigma) 7 days apart, AIA was induced ${ }^{29}$ by a single intra-articular injection (in $50 \mu \mathrm{L}$ saline) into the right knee 14 days later (day 0 ). Induction was either with $0.5 \mathrm{mg} \mathrm{mBSA}$ alone (15 rats, AIA) or $0.5 \mathrm{mg}$ mBSA+2.5 mM NBQX (Tocris) (15 rats, AIA $+\mathrm{NBQX}) .^{25}$ The main comparisons in this intervention study were between AIA and AIA+NBQX rats, but naive rats $(n=6)$ revealed effects of AIA. All measurements were taken at days 0 (baseline), 1, 2, 3, 4, 7, 9, 14 and 21, unless stated.

\section{Sample processing}

Human synovium, MTP and whole rat knees were fixed ( 2 days, $10 \%$ neutral buffered formalin, Sigma), decalcified $\left(4^{\circ} \mathrm{C}, 10 \%\right.$ EDTA, Fisher Scientific), paraffin embedded and coronally sectioned $(6 \mu \mathrm{m})$.

\section{Histology}

Consecutive sections from all human samples and nine AIA, nine AIA+NBQX and three naive rats (day 21) were stained with $\mathrm{H} \& \mathrm{E}$ (synovial inflammation), toluidine blue/safranin-O (cartilage and bone) or retained for immunohistochemistry.

Two independent observers blinded to treatment used published scoring systems to assess human $\mathrm{OA}^{30}$ and $\mathrm{rat}^{31}$ synovial inflammation and human MTP degradation, ${ }^{32}$ and a modified Mankin score for rat knee degradation (see online supplementary tables S1-S4). Average scores of two sections $\sim 500 \mu \mathrm{m}$ apart are presented for rats.

\section{Immunohistochemistry and TRAP staining}

GluRs were immunolocalised in sequential sections from human synovium, human MTP and rat knees (numbers as above) using antibodies to KA receptor-1 and AMPA receptor-2 (AMPAR2) (anti-KA1, anti-iGluR2; Abcam, see online supplementary methods). Sections underwent antigen retrieval $(1 \mathrm{mg} / \mathrm{mL}$ trypsin, Sigma), peroxidase blocking, overnight incubation $\left(4^{\circ} \mathrm{C}\right)$ with primary antibody and detection (Vectastain Elite ABC kit, nickel enhanced diaminobenzidine, Vector Laboratories). No primary antibody and IgG controls were included in every run (see online supplementary figure S1). Consecutive sections were tartrate resistant acid phosphatase (TRAP) stained ${ }^{33}$ (see online supplementary methods).

\section{Knee swelling}

Rat knee diameters were measured (mean of three readings per knee, Mitutoyo digital calliper, RS Components) and the difference between inflamed and contralateral knees presented.

\section{IL-6 ELISA}

Serum IL-6 concentrations were quantified from tail bleeds (six AIA, six AIA+NBQX, days 0, 1, 2, 3, 7, 14 and 21) (IL-6 Quantikine ELISA, R\&D Systems).

\section{Footprint scoring}

Following training, walking patterns were evaluated by hindfootprint analysis (six AIA, six AIA+NBQX, three naive rats). ${ }^{34}$ Paws were dipped in paint prior to free movement along a paper-lined walkway ( $1 \mathrm{~m}$ long, $10 \mathrm{~cm}$ wide). Three sets of six sequential steps were recorded per rat per day. Limb rotation, stride length and stance width were measured. ${ }^{34}$

\section{Imaging}

Whole rat knees (day 21) underwent X-ray and MRI. X-rays of both knees (nine AIA, nine AIA+NBQX, three naive) were obtained using a Kodak FX Pro In-vivo Imaging System (Advanced Molecular Vision). For MRI, both knees (six AIA, six AIA+NBQX) were imaged (Bruker Biospin Advance 9.4 T $(400 \mathrm{mHz})$ system, transmit-receive quadrature coil, RARE-MRI sequence: $\mathrm{TE}=1250 \mathrm{~ms}, \mathrm{TR}=24 \mathrm{~ms}, \mathrm{FA}=180^{\circ}$, matrix size $=580 \times 280 \times 280$, FOV $=58 \times 28 \times 28 \mathrm{~mm})$ and $3 \mathrm{D}$ datasets manipulated to obtain representative mid-sagittal planes of each knee (Analyze 10.0, Mayo Clinic, Rochester).

\section{mRNA expression}

Menisci, patella, femoral shaft (FS), femoral condyle (FC) and tibial plateaux (TP) were dissected from both knees (six AIA, six AIA+NBQX, day 21). Total RNA was extracted (TRIzol, Invitrogen), DNase treated (DNA-free, Ambion), 300 ng reverse transcribed (SuperScript III, Invitrogen; ribonuclease inhibitor and random primers, Promega) and messenger RNA (mRNA) expression quantified by RT-qPCR (SYBR Green JumpStart Taq ReadyMix, Sigma; Stratagene MX3000P) using intron-spanning primers (Primer 3) (see online supplementary table S5). ${ }^{20}$ Sequencing of cloned RT-PCR products confirmed primer specificity.

Standard curves for GluRs and IL-6 were generated from rat brain and spleen cDNAs, respectively, to confirm linearity $\left(\mathrm{R}^{2} \geq 0.95\right)$ and efficiency (90\%-110\%) for relative quantification. ${ }^{35}$

Absolute RT-qPCR (see online supplementary table S5) quantified osteoprotegerin (OPG), receptor activator of nuclear factor $\kappa-B$ ligand (RANKL), cathepsin $\mathrm{K}$ and collagen type $\mathrm{I}$ alpha (COL1A1) mRNA in FC and TP using standard curves $\left(10^{1}-10^{7}\right.$ copies $\left./ \mu \mathrm{L}\right)$ of RT-PCR products cloned in pGEM-T (Promega).

NormFinder identified the optimal combinations of reference genes (GAPDH, HPRT1, eEF2 and YWHAZ) for normalisation. $^{36}$

\section{Osteoblast assays}

The effects of NBQX $(200 \mu \mathrm{M})$ on cell number and mineralisation of human primary osteoblasts (HOBs) from OA total knee replacement bone (three patients) were assessed by an MTS assay (Promega) (12 replicates/patient) and Alizarin Red S staining $^{37}$ (20 days mineralising culture, four replicates/patient) respectively (see online supplementary methods). 


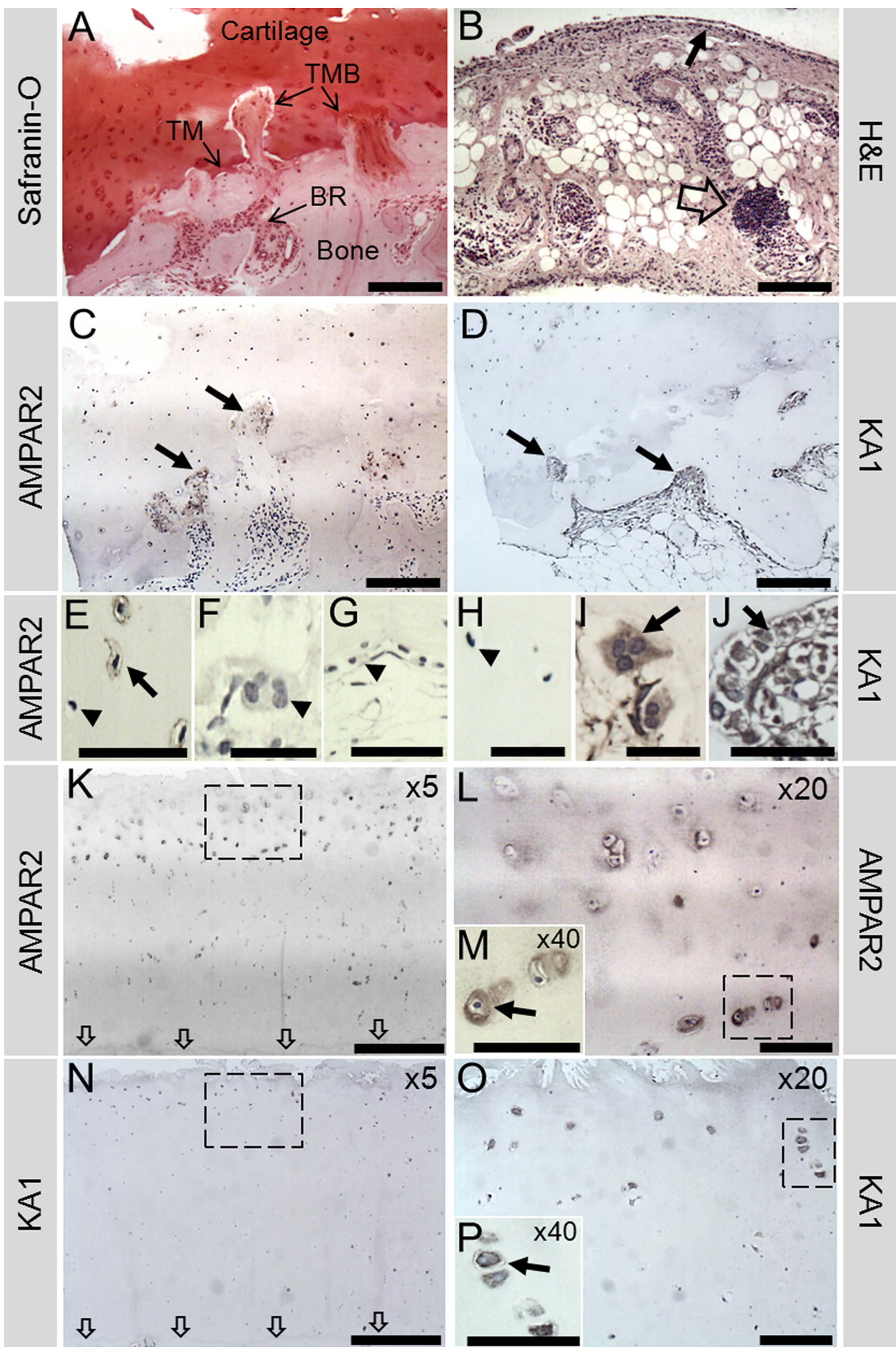

Figure 1 Representative human OA sample showing $\alpha$-amino-3-hydroxy-5-methyl-4-isoxazolepropionic acid receptor 2 (AMPAR2) and KA1 immunohistochemistry in the medial tibial plateaux (MTP). (A), (C) and (D) are all images from the same location in the outer MTP. (A) Safranin-0 stain reveals the architecture of the bone and cartilage, with extensive bone remodelling (BR) and breaching (TMB) of the tidemark (TM), which is almost completely lost. (B) Synovial tissue from the same patients showed evidence of inflammation indicated by perivascular lymphoid aggregates (open arrow) and a thickened synovial lining (small arrow). (C) AMPAR2 was localised to areas of remodelling, particularly to the TMB regions (arrows). (E) Osteocyte AMPAR2 staining was occasionally observed in small areas (arrow); however, many osteocytes remained negative (arrow head). No AMPAR2 staining was seen in osteoclasts (arrow head (F)) or bone lining cells (arrow head (G)) from normal areas of bone. (D) KA1 localised to remodelling bone (arrows), osteoclasts (arrow (I)) and osteoblasts (arrow (J)). No KA1 staining was seen in osteocytes (arrow head (H)). $(\mathrm{K}, \mathrm{L})$ AMPAR2 stained chondrocytes (arrow $(\mathrm{M})$ ) near the fibrillated cartilage surface down to the middle/deep zone interface, appearing strongest in the middle zone, with no staining near the TM (indicated by arrows). (N, 0) KA1 stained chondrocytes (arrow (P)) near the surface to the upper middle zone, with no staining in the deep zone. Corresponding negative controls (no primary antibody) and rabbit lgG controls were negative for KA1 and AMPAR2 (see online supplementary figure S1). Boxes indicate where higher power image was taken. Scale bars: (A-D), $200 \mu \mathrm{m} ;(E, G, J$, $\mathrm{M}, \mathrm{P}), 50 \mu \mathrm{m} ;(\mathrm{F}, \mathrm{H}, \mathrm{I}), 25 \mu \mathrm{m} ;(\mathrm{K}, \mathrm{N}), 500 \mu \mathrm{m} ;(\mathrm{L}, \mathrm{O}), 100 \mu \mathrm{m}$. 


\section{Statistics}

Using Minitab 16, data were tested for normality and equal variances prior to ANOVA (histological inflammation (Fisher's) and COL1A1, RANKL, OPG mRNA expression (Tukey-Kramer)) or general linear model two-way ANOVA (GluR mRNA expression (Tukey-Kramer)) with individual post hoc tests. Two sample $t$ tests were used for cell number. Non-parametric data used Kruskal-Wallis (footprints, histological joint degradation, IL-6 and cathepsin K mRNA expression) or Sheirer-Ray-Hare (knee swelling, joint compartment degradation) with Mann-Whitney post hoc tests. Means \pm SE of the mean (SEM) are presented.

\section{RESULTS}

\section{GluRs are expressed in human arthritis}

All patients showed cartilage fibrillation, tidemark breaches and proteoglycan loss, with OA MTP degradation scores ranging from 9 to 13 (figure 1A, see online supplementary figure S2). Synovial inflammation occurred in OA samples, with scores of 1-2 (figure 1B).
In OA, AMPAR2 localised to mononuclear cells (including some osteocytes) in areas of bone remodelling (figure 1C,E), but not osteoclasts (figure 1F). KA1 localised to remodelling bone (figure 1D), osteoclasts (figure 1I) and osteoblasts (figure $1 \mathrm{~J}$ ) but not to osteocytes (figure $1 \mathrm{H}$ ). Chondrocytes expressed both receptors, with more staining near the cartilage surface and none in the deep zone (figure $1 \mathrm{~K}-\mathrm{P}$ ). AMPAR2 and KA1 immunopositive chondrocytes were abundant in the middle section of MTP cartilage but less common in the severely degraded outer MTP cartilage (see online supplementary figure S2). AMPAR2 and KA1 staining in the bone localised mainly to remodelling bone in the outer segment of the MTP (see online supplementary figure S2). Similar patterns occurred in RA, with KA1 and AMPAR2 present in osteoclasts (see online supplementary figure S3).

\section{AIA and NBQX influence GluR expression}

KA1 and AMPAR2 proteins were expressed in chondrocytes and synovial lining cells (not shown) in all rats, and localised to remodelling bone in AIA and AIA+NBQX (figure 2).

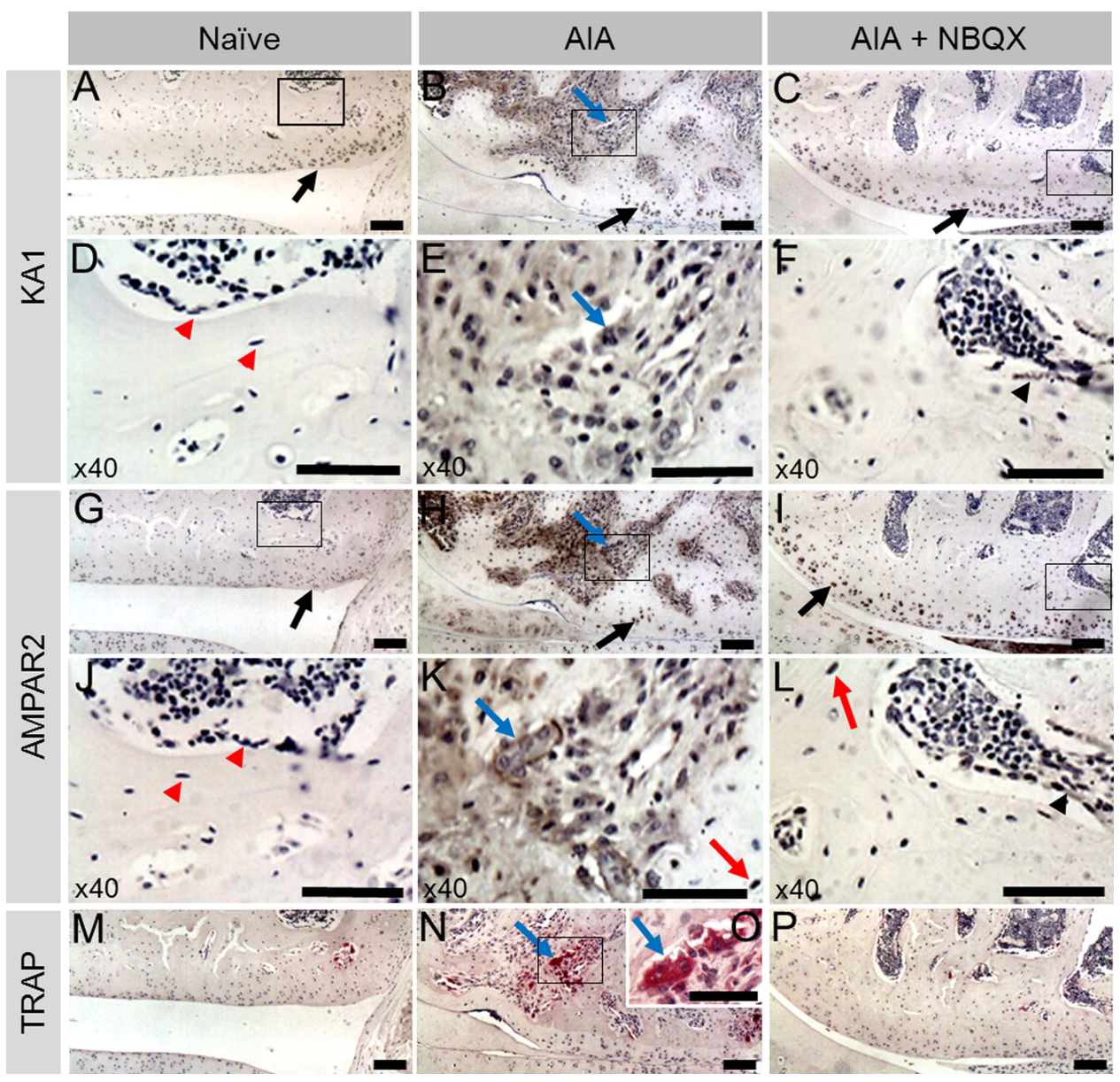

Figure 2 KA1 and $\alpha$-amino-3-hydroxy-5-methyl-4-isoxazolepropionic acid receptor 2 (AMPAR2) immunohistochemistry and tartrate resistant acid phosphatase (TRAP) staining in the lateral femoral condyle of naive, antigen-induced arthritis (AIA) and AIA+NBQX rats. Chondrocytes in all animals expressed KA1 and AMPAR2 (A-C, G-I, respectively, black arrows). Neither proteins localised to osteocytes or mononuclear bone cells (D, J, red arrow heads) in naive rats; however, in AIA and AIA+NBQX rats, AMPAR2 was expressed in osteocytes, mainly in areas of bone remodelling (K, $\mathrm{L}$, red arrow). In AIA rats, mononuclear bone cells and areas of bone remodelling stained intensely for KA1 and AMPAR2 (B, E, H, K). AIA+NBQX rats showed less bone remodelling and subsequently less staining of both proteins (C, F, I, L, black arrow heads). Abundant TRAP staining was found in AIA rats $(N)$ indicating the presence of more osteoclasts compared with naive $(M)$ and $A I A+N B Q X$ rats $(P)$. Consecutive sections showed expression of KA1 (E) and AMPAR2 (K) in TRAP positive osteoclasts (0) in AIA rats (blue arrows). Black boxes are shown at $\times 40$ in images underneath. (0) $\times 40$ Image of boxed area in N. Corresponding negative controls (no primary antibody) and rabbit IgG controls were negative for KA1 and AMPAR2 (see online supplementary figure S1). Scale bars: (A-C, G-I, M, N, P), $100 \mu \mathrm{m}$; (D-F, J-L, 0), $50 \mu \mathrm{m}$. 

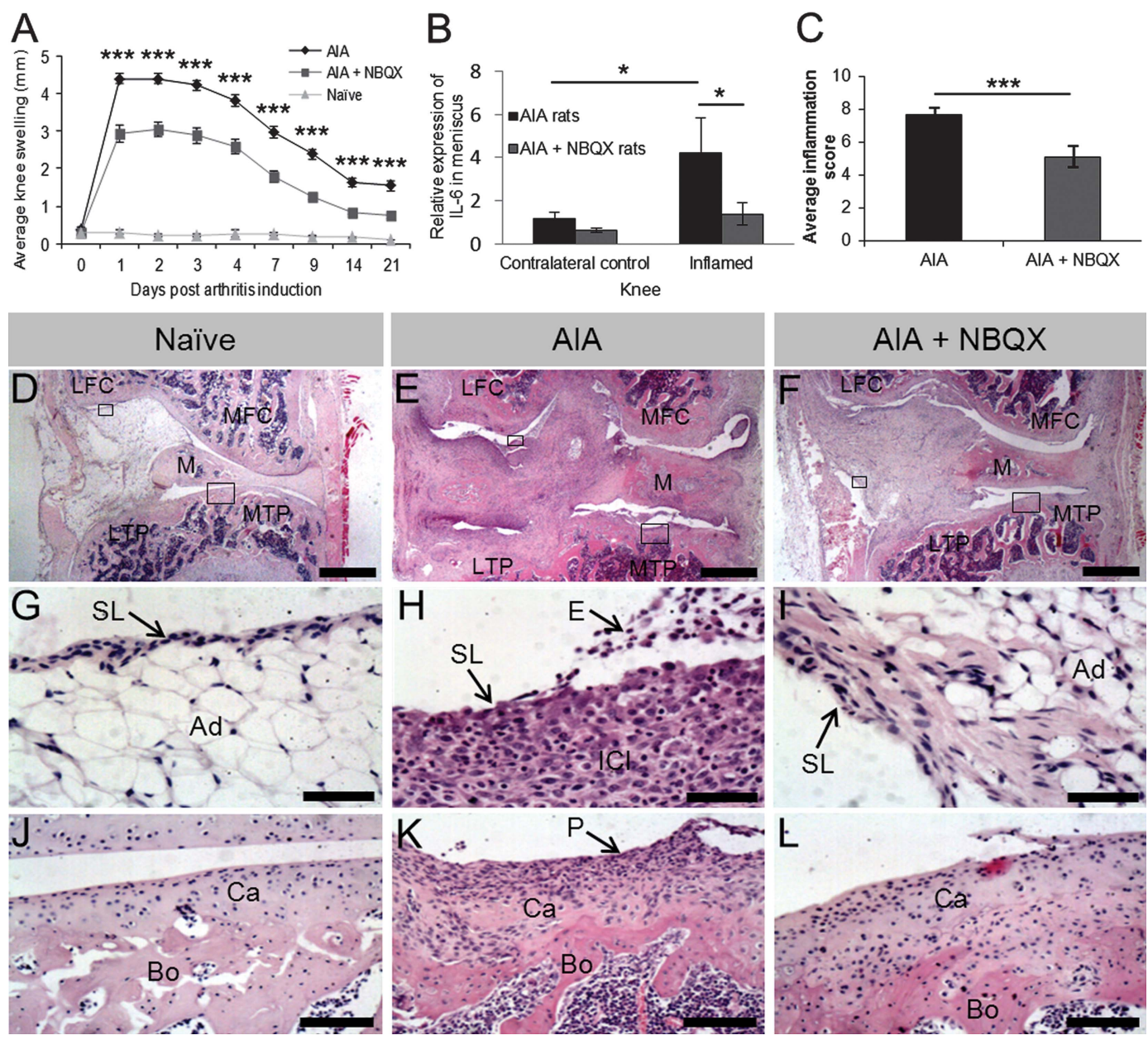

Figure 3 Swelling, synovial inflammation and IL-6 mRNA expression in knees from naive, antigen-induced arthritis (AIA) and AIA+NBQX rats culled on day 21. (A) Significantly less knee swelling was found in NBQX treated rats compared with AIA rats over 21 days $\left({ }^{* * *} p<0.001\right)$. (B) Significantly less IL-6 mRNA expression in the right inflamed knee was found in NBQX treated rats compared with AIA rats ( $\left.{ }^{*} p<0.05\right)$. (C) NBQX treated rats had a significantly lower inflammation score compared with AIA rats $\left({ }^{* *} p<0.001\right)$. (D) Naive animals had a normal synovial lining (SL) (G) which was 2-4 cells thick with adipose tissue (Ad) directly beneath. The articular surface $(\mathrm{J})$ consisted of a layer of smooth cartilage (Ca) over subchondral bone $(B))$. (E, F) Synovial hyperplasia (pannus (P)), exudate (E), inflammatory cell infiltrate (ICI) and articular surface degradation apparent in AIA rats $(H, K)$ was less severe in AIA+NBQX rats $(I, L)$. MTP, medial tibial plateaux; LTP, lateral tibial plateaux; MFC, medial femoral condyle; LFC, lateral femoral condyle; $M$, meniscus. Boxes in (D-F) indicate where images in (G-L) are from. Scale bars: (D-F), $1 \mathrm{~mm}$; (G-I), $50 \mu \mathrm{m}$; (J-L), $100 \mu \mathrm{m}$.

Osteocytes and other mononuclear cells in remodelling bone expressed AMPAR2 in AIA and AIA+NBQX (figure 2K,L). NBQX reduced the extent of remodelling, with an apparent reduction of GluR positive cells (figure 2). Neither AMPAR2 nor KA1 localised to mononuclear bone cells in naive animals (figure 2). TRAP positive osteoclasts in AIA coexpressed KA1 and AMPAR2 in consecutive sections (figure 2).

GluR transcripts (except GluR5 and NMDAR1) were detected in all rat joint tissues (see online supplementary figure S4). AIA and AIA+NBQX rats showed no differences in GluR mRNA expression, except for a fivefold increase in patella AMPAR3 in AIA that remained at contralateral control levels in AIA +NBQX $(p<0.05$, supplementary figure S4).

\section{NBQX reduces inflammation and IL- 6 expression}

Peak knee swelling following arthritis induction (day 1, 4.4 $\pm 0.14 \mathrm{~mm})$ was reduced in AIA+NBQX rats $(2.95 \pm 0.23 \mathrm{~mm}$, $33 \%$ reduction, $\mathrm{p}<0.001)$ and at all other time points $(\mathrm{p}<0.001$, figure $3 \mathrm{~A})$.
Serum IL-6 was undetectable in AIA samples $(<21 \mathrm{pg} / \mathrm{mL})$. However, at day 21, a threefold increase in meniscal IL-6 mRNA in the inflamed knee of AIA rats compared with the contralateral knee $(\mathrm{p}<0.05)$ remained at control levels in AIA $+\mathrm{NBQX}(\mathrm{p}<0.05$, figure $3 \mathrm{~B})$. IL-6 mRNA was not detected in FC, FS, TP and patella.

Synovial inflammation scores were reduced by NBQX treatment $(7.67 \pm 0.41$ vs $5.11 \pm 0.65, \mathrm{p}<0.001)$ (figure $3 \mathrm{C}$ ). Naive animals displayed normal synovial lining, 2-4 cells thick, with underlying adipose tissue, whereas AIA induced synovial hyperplasia, exudate and infiltrate that were reduced by NBQX treatment (figure 3D-L).

\section{NBQX restores weight bearing}

While AIA rats had no right hind-footprints on days 1 and 2 (figures 4A,B), NBQX restored weight bearing on these days, comparable with naive rats. Walking abnormalities occurred in AIA and AIA+NBQX rats, with greater foot rotation (figure 4B) and stance width (figure 4C) and shorter stride length (figure 4D) than naive rats $(\mathrm{p}<0.05)$. 
A

Naïve

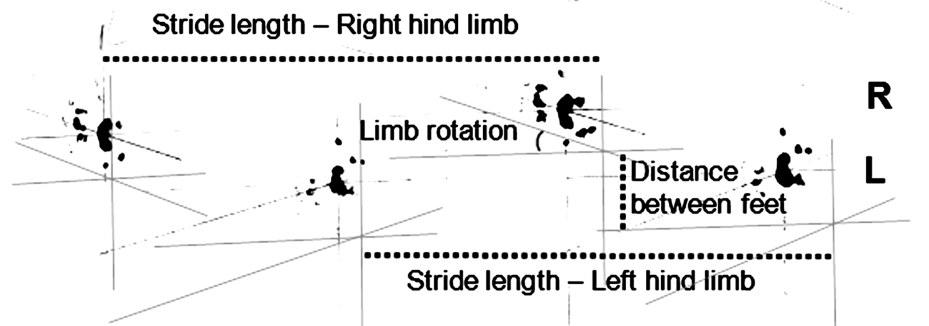

$\mathrm{AIA}+\mathrm{NBQX}$

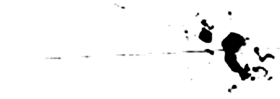

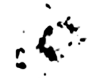
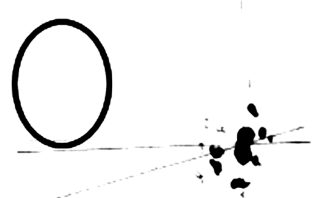

L
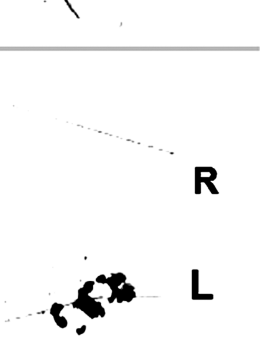
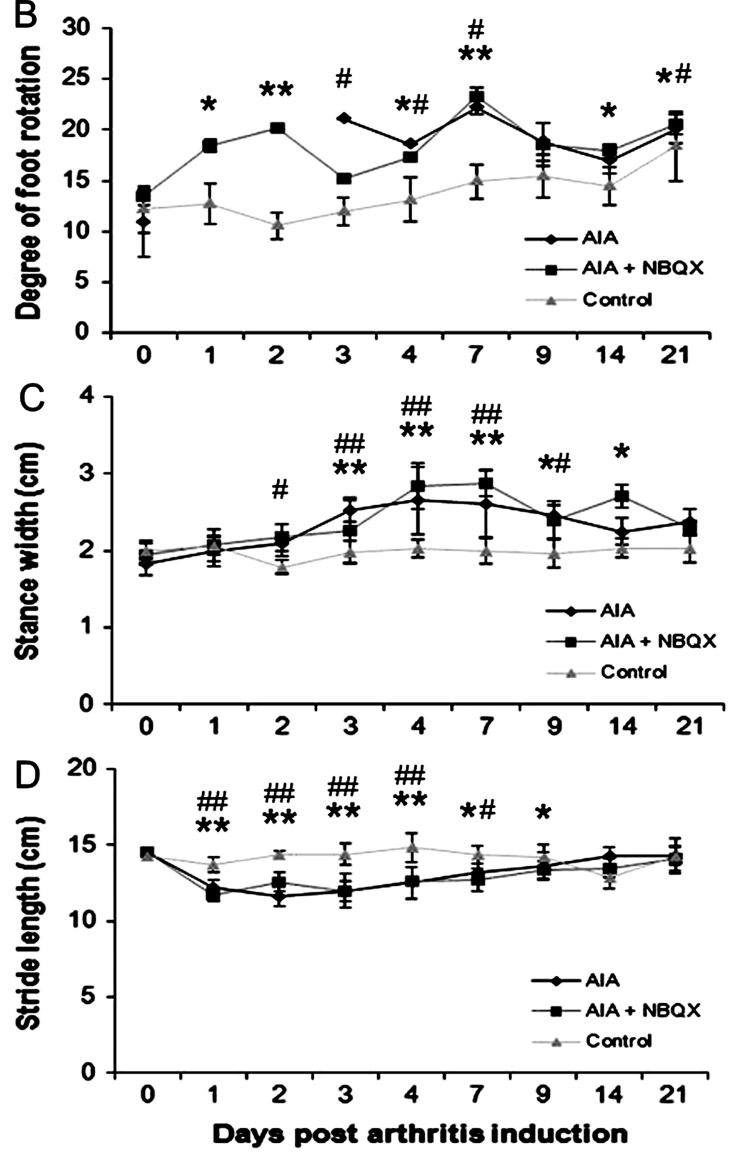

Figure 4 Footprint analysis of naive, antigen-induced arthritis (AIA) and AIA+NBQX rats. (A) Day 1 hindlimb footprints from the three experimental groups. AIA rats often lacked a right footprint (circled) whereas AIA+NBQX rats displayed a gait pattern resembling that of naive animals. Measurements of degree of foot rotation, stride length and stance width are indicated. (B-D) Analysis of foot rotation in the right inflamed limb (B), stance width (C) and stride length (D). (B) AIA and AIA+NBQX rats have a significantly greater degree of foot rotation in the right limb compared with naive rats. On days 1 and 2, AIA rats were unable to weight bear and therefore lack data points. Stance width was increased (C) and stride length decreased (D) in AIA and AIA+NBQX rats compared with naive. ${ }^{*} p<0.05,{ }^{* *} p<0.001 \mathrm{AIA}+\mathrm{NBQX}$ compared with naive; ${ }^{\#} p<0.05$, $\#$ \# $<0.001$ AlA compared with naive.

\section{NBQX reduces joint degradation}

NBQX treatment reduced cartilage and bone pathology (figure 5). AIA caused loss of cartilage and substantial subchondral bone remodelling, whereas NBQX treated knees resembled those from naive rats, except for remodelling at the outer edges (figure $5 \mathrm{~A}$ ). NBQX reduced AIA severity score $(39.3 \pm 4.6)$ by $27 \%(28.8 \pm 1.7$, $\mathrm{p}<0.001)$ although not to naive values $(11.7 \pm 2.7, \mathrm{p}<0.001)$ (figure 5B).

While severity scores did not vary significantly across joint quadrants (MTP, lateral TP, medial FC, lateral FC), scores were lower in the whole FC following NBQX treatment $(20.9 \pm 2.99$ (AIA) to $12.7 \pm 0.85$ (AIA+NBQX), $\mathrm{p}<0.01$, figure 5C). NBQX lowered each score component, showing the greatest effect in bone (figure 5D, see online supplementary table S6). Severe bone erosions and synovial inflammation in AIA revealed by $\mathrm{x}$-ray (figure $6 \mathrm{~A}-\mathrm{C}$ ) and MRI (figure 6D-F) were attenuated by NBQX treatment.

\section{NBQX affects bone markers}

Threefold increases in cathepsin $\mathrm{K}$ mRNA (pooled FC and TP) in AIA compared with contralateral control knees $(\mathrm{p}<0.01)$ was halved by NBQX $(p<0.05)$, but not restored to control values $(\mathrm{p}<0.05$, figure 6G). COL1A1 expression was increased in AIA $(\mathrm{p}<0.001)$ and AIA+NBQX $(\mathrm{p}<0.05)$ compared with contralateral controls (figure $6 \mathrm{H}$ ). Increased RANKL mRNA expression $(p<0.05)$ and RANKL to OPG ratios $(p<0.01)$ in AIA compared with contralateral controls were prevented by NBQX treatment (figure 6I,K). Neither AIA nor AIA+NBQX affected OPG mRNA expression (figure 6J).

\section{NBQX reduces $H O B$ number and mineralisation}

NBQX treatment reduced $\mathrm{HOB}$ number at days 2 and 5 $(\mathrm{p}<0.001)$ and prevented mineralisation in all cultures (see online supplementary figure S5).

\section{DISCUSSION}

To determine whether glutamatergic signalling influences local inflammatory processes underlying arthritic pathologies, we investigated synovial inflammation and AMPA/KA GluR expression in human OA, RA and rat AIA, and determined whether AMPA/KA GluR antagonists affect AIA pathology. Characteristic synovial inflammation occurred in all arthritis patients. ${ }^{30} 3839$ AMPA and KA GluRs were expressed in inflamed synovium, and diseased areas of bone and cartilage in human arthritic tissue and rat AIA. A single intra-articular NBQX injection profoundly reduced joint pathology in AIA, reducing knee swelling by 33\%, histological synovial inflammation scores by $34 \%$ and degeneration scores by $27 \%$. The protection offered by NBQX exceeded 
A

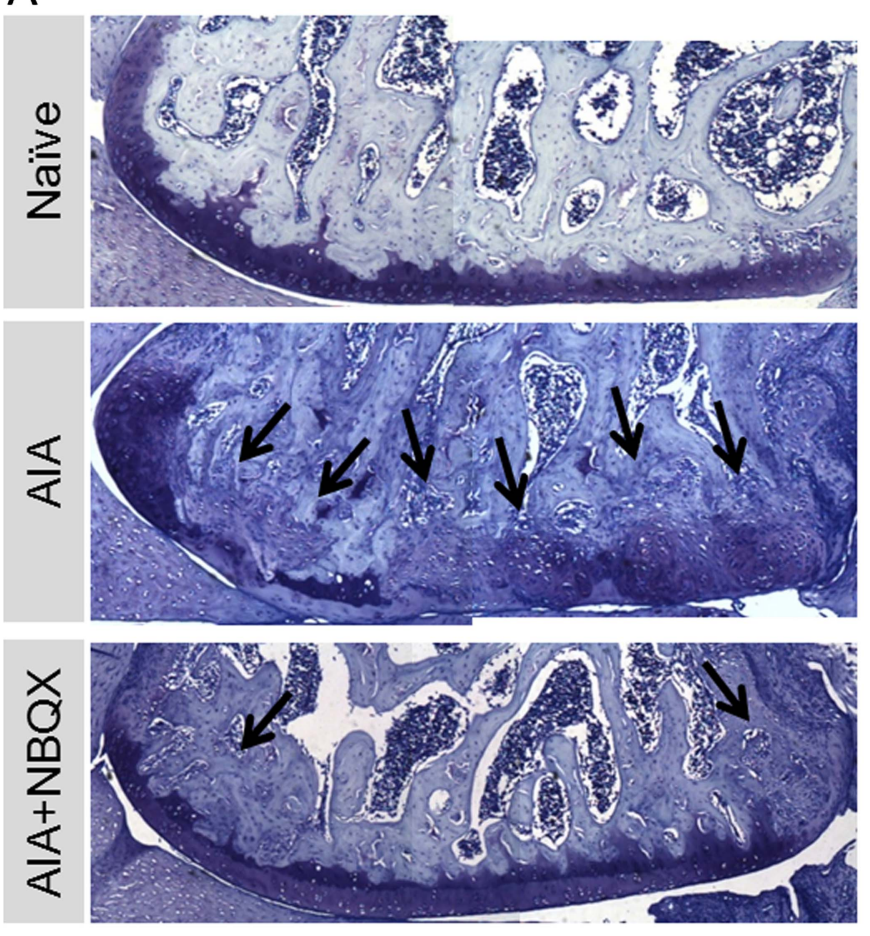

B
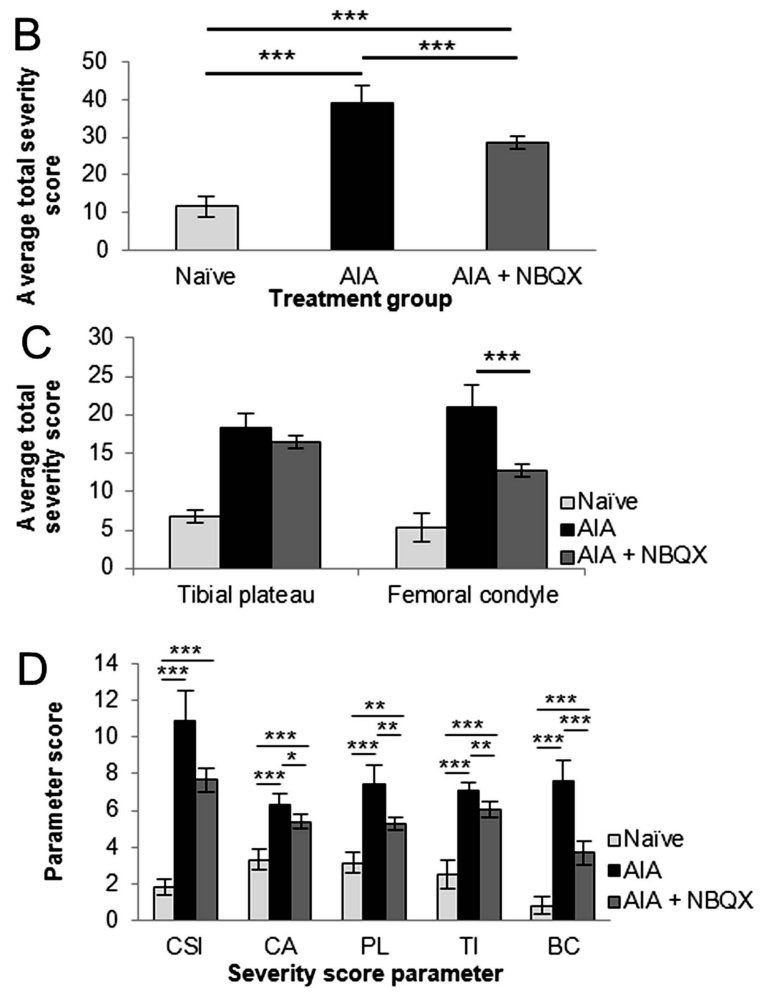

Figure 5 Joint degradation and remodelling in naive, antigen-induced arthritis (AIA) and AIA+NBQX rats on day 21. (A) Representative toluidine blue stains of the lateral femoral condyle. $(A, B) A I A+N B Q X$ rats displayed less severe cartilage and bone pathology scores compared with AIA rats $(p<0.001)$. (C) AIA+NBQX rats showed a significantly lower joint severity score in the femoral condyle compared with AIA rats ( $p<0.001)$. Abundant bone remodelling in AIA rats, indicated by toluidine blue staining $(A)$, was significantly reduced in AIA+NBQX rats (arrows, $p<0.001)(A, D(B C$ parameter)). (D) Chondrocyte appearance, proteoglycan loss and tidemark integrity scores were also lower in AIA+NBQX compared with AIA rats $(p<0.01)$. CSI, cartilage surface integrity; $C A$, chondrocyte appearance; $\mathrm{PL}$, proteoglycan loss; $\mathrm{TI}$, tidemark integrity; $\mathrm{BC}$, bone changes. ${ }^{*} \mathrm{p}<0.05$, ${ }^{* *} \mathrm{p}<0.01,{ }^{* * *} \mathrm{p}<0.001$.

that of etanercept, infliximab and methotrexate in the same model. A single intra-articular injection of methotrexate at the time of induction did not reduce swelling or degeneration, and although liposomally conjugated methotrexate reduced knee swelling by $39 \%$ on day 1 , long-term effects are unreported. ${ }^{29}$ Six intraperitoneal injections of etanercept and infliximab had milder effects on swelling than NBQX ( 20\% reduction, days 17 ), and no effect on joint pathology at day 21 in rat AIA. ${ }^{40}$ Continuous administration of etanercept (intrathecal) ${ }^{41}$ and leflunomide (oral) ${ }^{42}$ was required to reduce joint pathology in rat AIA. Thus, NBQX treatment in the AIA model is more effective than equivalent administration of approved drugs.

This is the first report to demonstrate localisation of GluRs to bone, cartilage and synovial cells in human OA and RA tissue. This is particularly important for OA as it is a common disease, with limited therapeutic options, where current trials are testing efficacy of anti-inflammatory treatments. ${ }^{43}{ }^{44}$ In human OA and RA, AMPAR2 localised to mononuclear bone cells, including osteocytes, and KA1 to osteoclasts and osteoblasts but not osteocytes in remodelling bone. Similarly, in rat AIA, mononuclear cells and TRAP stained osteoclasts in remodelling bone expressed AMPAR2 and KA1, consistent with the effects of these iGluRs on osteoblast ${ }^{45}$ and osteoclast activities. ${ }^{46}$ NBQX treatment in AIA reduced bone remodelling and therefore GluR abundance. Rodent osteoblasts, osteocytes and osteoclasts express AMPAR2 protein, and osteoblasts express KA1, ${ }^{16}$ but there have been no reports in human bone cells. AMPAR2 was not detected in osteocytes in naive animals, consistent with previous reports, ${ }^{46}$ but was expressed in AIA osteocytes.
AMPAR2 and KA1 were expressed by chondrocytes in naive, AIA and AIA+NBQX rats, and in human OA and RA tissue, where GluRs were abundant near the surface and towards the mid-zone. Chondrocytes release glutamate and express $\mathrm{AMPA}^{47}$ and NMDA GluRs. ${ }^{18}$ NMDA GluR antagonists decrease proliferation and inhibit IL-1 $\beta$ induced increases in cyclooxygenase-2, IL-6 and MMP3 mRNA expression in chondrocytes. ${ }^{18}$ However, KA GluR expression and the role of AMPA/KA GluRs have not been reported in chondrocytes.

Our observation that NBQX treatment reduced knee swelling and synovial inflammation over 21 days is the first to show an effect of AMPA/KA GluR antagonists on swelling and long-lasting anti-inflammatory effects of any GluR antagonist after a single injection. A study targeting all iGluRs with a single intra-articular injection in rat CFA arthritis only reported short-term reduction of swelling. ${ }^{27}$ An NMDA GluR antagonist had long-term effects on paw synovitis in mouse CIA, but this required 12-hourly, intraperitoneal injections. ${ }^{21}$ The anti-inflammatory effects of NBQX may be mediated by IL- $6 .{ }^{20}$ Although serum IL- 6 concentrations were too low to quantify, ${ }^{48}{ }^{49}$ increased meniscal IL- 6 mRNA expression in AIA was reduced by NBQX treatment, suggesting that bone, marrow and/or cartilage cells ${ }^{50}$ in the meniscus may respond to glutamate to produce IL-6..$^{51-53}$

NBQX treatment restored weight bearing over 2 days following AIA induction, probably reflecting reduced pain. ${ }^{54}$ Previous studies found that injection of MK801 (NMDAR antagonist) or NBQX into the rat knee inhibits arthritis pain for $24 \mathrm{~h},{ }^{25}$ a single intra-articular injection of combined NMDAR and AMPA/KA GluR antagonists alleviates allodynia over 3 days ${ }^{27}$ 


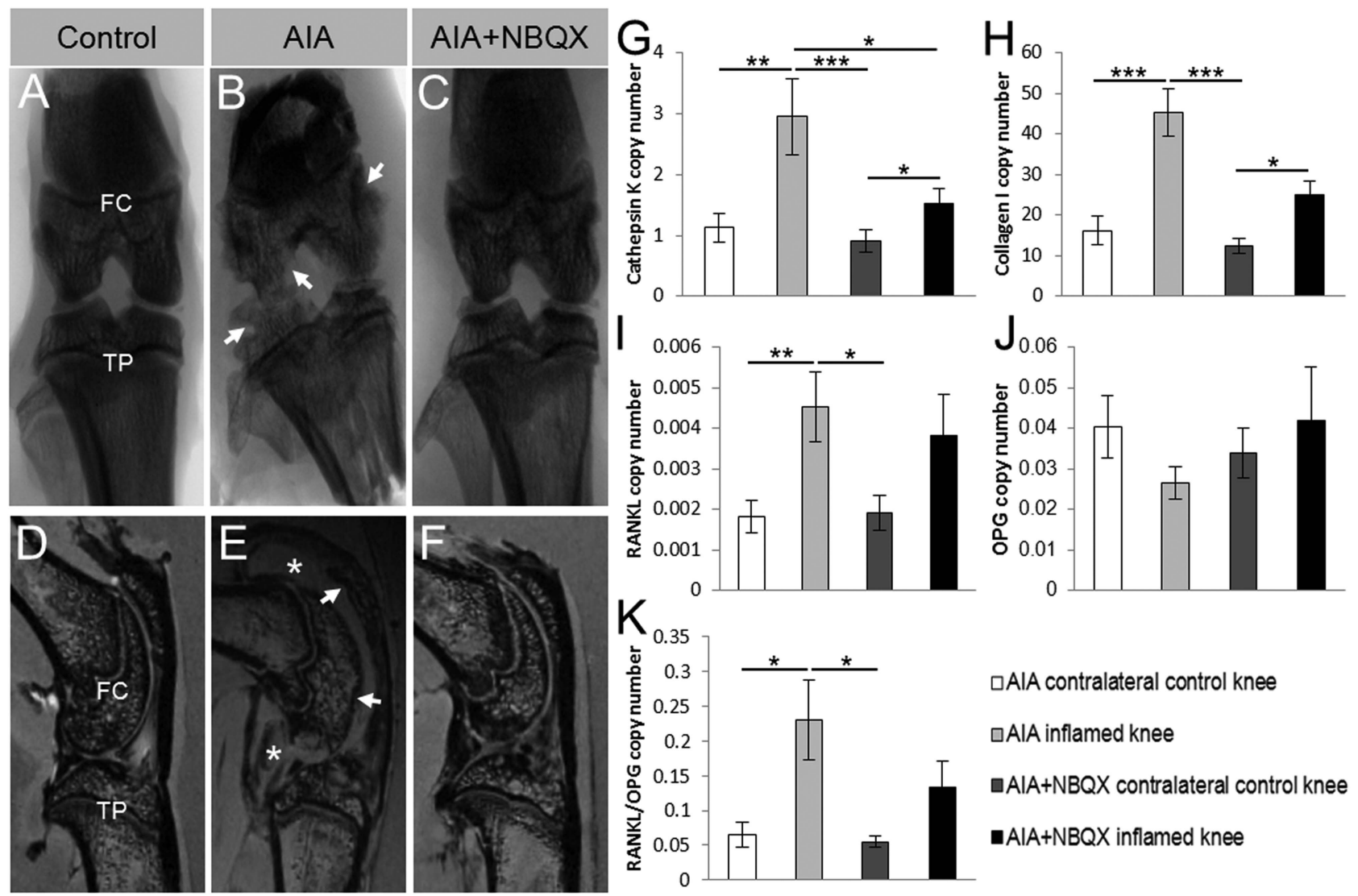

Figure 6 Macroscopic joint pathology and bone phenotype mRNA expression in antigen-induced arthritis (AIA) and AIA+NBQX inflamed and contralateral control rat knees. $(A-C)$ Representative $x$-ray images show severe erosions in the tibial plateaux and femoral condyle in AIA rats (arrows, (B)). AIA+NBQX rats displayed a much smoother joint surface (C) resembling that seen in the contralateral control knee (A). (D-F) Representative MRIs confirm the erosions seen in X-rays (arrows), and also show the presence of severe synovial inflammation at day 21 (stars) in AIA rats (E). Synovial inflammation in AIA+NBQX knees was greatly reduced, as was joint erosion (F). FC, femoral condyle; TP, tibial plateaux. (G-K) Cathepsin $\mathrm{K}$, collagen I, receptor activator of nuclear factor $\kappa-B$ ligand (RANKL) and the RANKL to osteoprotegerin (OPG) ratio $\mathrm{mRNA}$ expression levels were significantly increased in the AIA inflamed knee compared with the AIA and AIA+NBQX contralateral control knees. (G, H) Cathepsin $K$ and collagen I mRNA expression was also significantly increased in inflamed AIA+NBQX knees compared with the AIA+NBQX contralateral control. (G) A significant reduction in cathepsin K mRNA expression was found in AIA+NBQX inflamed knees compared with AIA inflamed knees. (J) There were no differences in OPG expression. ${ }^{*} \mathrm{p}<0.05,{ }^{* *} \mathrm{p}<0.01,{ }^{* * *} \mathrm{p}<0.001$.

and repeated injection of AMPA GluR antagonists $(0-23 \mathrm{~h})$ following CFA arthritis alleviates inflammatory pain. ${ }^{26}$ However, our data are the first to demonstrate 2-day restoration of joint loading from a single intra-articular treatment of one GluR antagonist. This body of evidence indicates that peripheral inhibition of AMPA/KA GluRs reduces pain in arthritis.

This is the first study to show that a single intra-articular injection of any GluR antagonist alleviates cartilage and bone destruction in arthritis. A single intra-articular injection of combined iGluR antagonists did not affect cartilage erosion in CFA arthritis. ${ }^{27}$ While memantine (NMDAR antagonist) alleviated synovitis and joint pathology in CIA, continual 12-hourly intraperitoneal administration of the drug was necessary. ${ }^{21}$

Since AMPA/KA GluRs localised to remodelling bone in human OA, RA and rat AIA, we quantified GluR and bone cell mRNAs in joint tissues. Increased AMPAR3 mRNA expression in AIA patella was restored to normal by NBQX, and coincided with increased mRNAs reflecting osteoclast activation (RANKL), bone resorption (Cathepsin K) and bone formation (COL1A1). Cathepsin $\mathrm{K}$ and RANKL mRNA levels and RANKL to OPG ratios were reduced by NBQX. AMPA increases bone formation and mineralisation, ${ }^{45}$ whereas AMPAR antagonists reduce bone mass, ${ }^{55}$ inhibiting osteoblast activity and mineralisation. ${ }^{45}$
Consistent with this, NBQX reduced cell number and prevented mineralisation in $\mathrm{HOBs}$ from $\mathrm{OA}$ patients. Thus, the protective effect of NBQX in AIA may reflect inhibition of osteoblast activity associated with reduced RANKL mediated activation of osteoclasts. However, NBQX may also target AMPA and KA GluRs expressed by synoviocytes ${ }^{56}$ and chondrocytes ${ }^{57}$ to regulate RANKL or directly inhibit osteoclast activity. ${ }^{46}$

In conclusion, a single intra-articular injection of NBQX alleviated inflammation, pain and joint degeneration in rat AIA. Thus, AMPA/KA GluR antagonists have potential to alleviate multiple symptoms in any form of arthritis where local inflammatory processes are involved. GluR antagonists, tolerated in humans, ${ }^{58-60}$ and which do not cross the blood-brain barrier, ${ }^{58} 61$ are a timely potential therapeutic for modulating glutamatergic signalling in joints to treat arthritis.

Acknowledgements We are grateful to Derek Scarborough, Mari Nowell, Alex Klein, Eleri Jones, Samantha Lai-Morrice, Carole Elford, Helen Hodgson, Andrea Longman, Chris Wilson and Karen Brakspear for their contributions to this work.

Contributors The corresponding author confirms that all the individuals listed as authors fulfil the uniform authorship credit requirements for manuscripts submitted to medical journals, that is, that they all contributed to the manuscript based on (1) substantial contributions to conception and design, acquisition of data, or analysis 
and interpretation of data; (2) drafting the article or revising it critically for important intellectual content; and (3) final approval of the version to be published.

Funding This work within the Arthritis Research UK Biomechanics and Bioengineering Centre was funded by Arthritis Research UK and Cardiff University, and supported by National Institute for Social Care and Health Research Clinical Research Centre (NISCHR CRC).

Competing interests None.

Ethics approval Research Ethics Committee for Wales.

Provenance and peer review Not commissioned; externally peer reviewed.

Open Access This is an Open Access article distributed in accordance with the Creative Commons Attribution Non Commercial (CC BY-NC 3.0) license, which permits others to distribute, remix, adapt, build upon this work non-commercially, and license their derivative works on different terms, provided the original work is properly cited and the use is non-commercial. See: http://creativecommons.org/ licenses/by-nc/3.0/

\section{REFERENCES}

1 Bonnet CS, Walsh DA. Osteoarthritis, angiogenesis and inflammation. Rheumatology (Oxford) 2005;44:7-16.

2 Ashraf S, Walsh DA. Angiogenesis in osteoarthritis. Curr Opin Rheumatol 2008;20:573-80.

3 Berenbaum F. Osteoarthritis as an inflammatory disease (osteoarthritis is not osteoarthrosis!). Osteoarthritis Cartilage 2013;21:16-21.

4 Colmegna I, Ohata BR, Menard HA. Current understanding of rheumatoid arthritis therapy. Clin Pharmacol Ther 2012;91:607-20.

5 Cheng DS, Visco CJ. Pharmaceutical therapy for osteoarthritis. Pm R 2012;4(5 suppl):S82-8.

6 Hameed F, Ihm J. Injectable medications for osteoarthritis. Pm R 2012;4(5 suppl): S75-81.

7 Verbruggen $G$, Wittoek $R$, Vander Cruyssen $B$, et al. Tumour necrosis factor blockade for the treatment of erosive osteoarthritis of the interphalangeal finger joints: a double blind, randomised trial on structure modification. Ann Rheum Dis 2012;71:891-8.

8 Chevalier X, Goupille P, Beaulieu AD, et al. Intraarticular injection of anakinra in osteoarthritis of the knee: a multicenter, randomized, double-blind, placebo-controlled study. Arthritis Rheum 2009;61:344-52.

9 Maksymowych WP, Russell AS, Chiu P, et al. Targeting tumour necrosis factor alleviates signs and symptoms of inflammatory osteoarthritis of the knee. Arthritis Res Ther 2012;14:R206.

10 McNearney T, Speegle D, Lawand N, et al. Excitatory amino acid profiles of synovial fluid from patients with arthritis. J Rheumatol 2000;27:739-45.

11 Lawand NB, McNearney T, Westlund KN. Amino acid release into the knee joint: key role in nociception and inflammation. Pain 2000;86:69-74

12 Jean $\mathrm{YH}$, Wen ZH, Chang YC, et al. Increased concentrations of neuro-excitatory amino acids in rat anterior cruciate ligament-transected knee joint dialysates: a microdialysis study. J Orthop Res 2005;23:569-75.

13 McNearney T, Baethge BA, Cao S, et al. Excitatory amino acids, TNF-alpha, and chemokine levels in synovial fluids of patients with active arthropathies. Clin Exp Immunol 2004;137:621-7.

14 McNearney TA, Ma Y, Chen Y, et al. A peripheral neuroimmune link: glutamate agonists upregulate NMDA NR1 receptor mRNA and protein, vimentin, TNF-alpha, and RANTES in cultured human synoviocytes. Am J Physiol Regul Integr Comp Physiol 2010;298:R584-98.

15 Hinoi $E$, Yoneda $Y$. Possible involvement of glutamatergic signaling machineries in pathophysiology of rheumatoid arthritis. J Pharmacol Sci 2011;116:248-56.

16 Brakspear KS, Mason DJ. Glutamate signaling in bone. Front Endocrinol (Lausanne) 2012;3:97.

17 Skerry TM. The role of glutamate in the regulation of bone mass and architecture. J Musculoskelet Neuronal Interact 2008;8:166-73.

18 Piepoli T, Mennuni L, Zerbi S, et al. Glutamate signaling in chondrocytes and the potential involvement of NMDA receptors in cell proliferation and inflammatory gene expression. Osteoarthritis Cartilage 2009;17:1076-83.

19 Morimoto R, Uehara S, Yatsushiro S, et al. Secretion of L-glutamate from osteoclasts through transcytosis. Embo J 2006;25:4175-86.

20 Flood S, Parri R, Williams A, et al. Modulation of interleukin-6 and matrix metalloproteinase 2 expression in human fibroblast-like synoviocytes by functional ionotropic glutamate receptors. Arthritis Rheum 2007;56: 2523-34.

21 Lindblad SS, Mydel P, Hellvard A, et al. The N-methyl-d-aspartic acid receptor antagonist memantine ameliorates and delays the development of arthritis by enhancing regulatory T cells. Neurosignals 2012;20:61-71.

22 Miller KE, Hoffman EM, Sutharshan M, et al. Glutamate pharmacology and metabolism in peripheral primary afferents: physiological and pathophysiological mechanisms. Pharmacol Ther 2011;130:283-309.
23 Parada-Turska J, Rzeski W, Majdan M, et al. Effect of glutamate receptor antagonists and antirheumatic drugs on proliferation of synoviocytes in vitro. Eur $J$ Pharmacol 2006;535:95-7.

24 Parada-Turska J, Rzeski W, Zgrajka W, et al. Kynurenic acid, an endogenous constituent of rheumatoid arthritis synovial fluid, inhibits proliferation of synoviocytes in vitro. Rheumatol Int 2006;26:422-6.

25 Zhang GH, Yoon YW, Lee KS, et al. The glutamatergic N-methyl-D-aspartate and non-N-methyl-D-aspartate receptors in the joint contribute to the induction, but not maintenance, of arthritic pain in rats. Neurosci Lett 2003;351:177-80.

26 Gangadharan V, Wang R, Ulzhofer B, et al. Peripheral calcium-permeable AMPA receptors regulate chronic inflammatory pain in mice. J Clin Invest 2011:121:1608-23.

27 Lam FF, Ng ES. Substance P and glutamate receptor antagonists improve the anti-arthritic actions of dexamethasone in rats. Br J Pharmacol 2010;159:958-69.

28 Alonzi T, Fattori E, Lazzaro D, et al. Interleukin 6 is required for the development of collagen-induced arthritis. J Exp Med 1998;187:461-8.

29 Williams AS, Topley N, Dojcinov S, et al. Amelioration of rat antigen-induced arthritis by liposomally conjugated methotrexate is accompanied by down-regulation of cytokine mRNA expression. Rheumatology (Oxford) 2001;40:375-83.

30 Haywood L, McWilliams DF, Pearson Cl, et al. Inflammation and angiogenesis in osteoarthritis. Arthritis Rheum 2003;48:2173-7.

31 Nowell MA, Williams AS, Carty SA, et al. Therapeutic targeting of IL-6 trans signaling counteracts STAT3 control of experimental inflammatory arthritis. J Immunol 2009;182:613-22.

32 Walsh DA, Bonnet CS, Turner EL, et al. Angiogenesis in the synovium and the osteochondral junction in osteoarthritis. Osteoarthritis Cartilage 2007;15:743-51.

33 Wang N, Robaye B, Agrawal A, et al. Reduced bone turnover in mice lacking the P2Y(13) receptor of ADP. Mol Endocrinol 2012;26:142-52.

34 Klein A, Wessolleck J, Papazoglou A, et al. Walking pattern analysis after unilateral 6-OHDA lesion and transplantation of foetal dopaminergic progenitor cells in rats. Behav Brain Res 2009;199:317-25.

35 Pfaffl MW. A new mathematical model for relative quantification in real-time RT-PCR. Nucleic Acids Res 2001;29:e45.

36 Andersen $C_{L}$, Jensen JL, Orntoft TF. Normalization of real-time quantitative reverse transcription-PCR data: a model-based variance estimation approach to identify genes suited for normalization, applied to bladder and colon cancer data sets. Cancer Res 2004;64:5245-50.

37 Gharibi B, Abraham AA, Ham J, et al. Adenosine receptor subtype expression and activation influence the differentiation of mesenchymal stem cells to osteoblasts and adipocytes. J Bone Miner Res 2011;26:2112-24.

38 Revell PA, Mayston V, Lalor P, et al. The synovial membrane in osteoarthritis: a histological study including the characterisation of the cellular infiltrate present in inflammatory osteoarthritis using monoclonal antibodies. Ann Rheum Dis 1988;47:300-7.

39 Lee DM, Weinblatt ME. Rheumatoid arthritis. Lancet 2001;358:903-11.

40 Boettger MK, Hensellek S, Richter F, et al. Antinociceptive effects of tumor necrosis factor alpha neutralization in a rat model of antigen-induced arthritis: evidence of a neuronal target. Arthritis Rheum 2008;58:2368-78.

41 Boettger MK, Weber K, Grossmann D, et al. Spinal tumor necrosis factor alpha neutralization reduces peripheral inflammation and hyperalgesia and suppresses autonomic responses in experimental arthritis: a role for spinal tumor necrosis factor alpha during induction and maintenance of peripheral inflammation. Arthritis Rheum 2010:62:1308-18.

42 Coulthard LG, Costello J, Robinson B, et al. Comparative efficacy of a secretory phospholipase $\mathrm{A} 2$ inhibitor with conventional anti-inflammatory agents in a rat model of antigen-induced arthritis. Arthritis Res Ther 2011;13:R42.

43 Kraus VB, Birmingham J, Stabler TV, et al. Effects of intraarticular IL1-Ra for acute anterior cruciate ligament knee injury: a randomized controlled pilot trial (NCT00332254). Osteoarthritis Cartilage 2012;20:271-8.

44 Cohen SB, Proudman S, Kivitz AJ, et al. A randomized, double-blind study of AMG 108 (a fully human monoclonal antibody to IL-1R1) in patients with osteoarthritis of the knee. Arthritis Res Ther 2011;13:R125.

45 Lin TH, Yang RS, Tang CH, et al. Regulation of the maturation of osteoblasts and osteoclastogenesis by glutamate. Eur J Pharmacol 2008;589:37-44.

46 Szczesniak AM, Gilbert RW, Mukhida M, et al. Mechanical loading modulates glutamate receptor subunit expression in bone. Bone 2005;37:63-73.

47 Wang L, Hinoi E, Takemori A, et al. Release of endogenous glutamate by AMPA receptors expressed in cultured rat costal chondrocytes. Biol Pharm Bull 2005;28:990-3

48 Roth A, Mollenhauer J, Wagner A, et al. Intra-articular injections of high-molecular-weight hyaluronic acid have biphasic effects on joint inflammation and destruction in rat antigen-induced arthritis. Arthritis Res Ther 2005;7:R677-86.

49 Nissler K, Pohlers D, Huckel M, et al. Anti-CD4 monoclonal antibody treatment in acute and early chronic antigen induced arthritis: influence on macrophage activation. Ann Rheum Dis 2004;63:1470-7.

50 Pedersen HE. The ossicles of the semilunar cartilages of rodents. Anat Rec 1949:105:1-9. 
51 Guerne PA, Carson DA, Lotz M. IL-6 production by human articular chondrocytes Modulation of its synthesis by cytokines, growth factors, and hormones in vitro. $J$ Immunol 1990;144:499-505.

52 Evans BA, Elford C, Pexa A, et al. Human osteoblast precursors produce extracellular adenosine, which modulates their secretion of IL- 6 and osteoprotegerin. J Bone Miner Res 2006;21:228-36.

53 Cheung J, Mak YT, Papaioannou S, et al. Interleukin-6 (IL-6), IL-1, receptor activator of nuclear factor kappaB ligand (RANKL) and osteoprotegerin production by human osteoblastic cells: comparison of the effects of 17-beta oestradiol and raloxifene. J Endocrinol 2003;177:423-33.

54 Min SS, Han JS, Kim Yl, et al. A novel method for convenient assessment of arthritic pain in voluntarily walking rats. Neurosci Lett 2001;308:95-8.

55 Burford JH, Perrien DS, Horner A, et al. Glutamate signalling regulates skeletogenesis and bone growth. J Bone Miner Res 2004;19:S212-S3.
56 Byron $C R$, Barger $A M$, Stewart $A A$, et al. In vitro expression of receptor activator of nuclear factor-kappaB ligand and osteoprotegerin in cultured equine articular cells. Am J Vet Res 2010;71:615-22.

57 Carda C, Silvestrini G, Gomez de Ferraris ME, et al. Osteoprotegerin (OPG) and RANKL expression and distribution in developing human craniomandibular joint. Tissue Cell 2005:37:247-55.

58 Herrling P. ed. Excitatory amino acids: clinical results with antagonists. Academic Press, 1997.

59 Dal D, Tetik O, Altunkaya $\mathrm{H}$, et al. The efficacy of intra-articular ketamine for postoperative analgesia in outpatient arthroscopic surgery. Arthroscopy 2004;20:300-5.

60 Ingwersen SH, Ohrstrom JK, Petersen $\mathrm{P}$, et al. Human Pharmacokinetics of the Neuroprotective Agent NBQX. Am J Ther 1994;1:296-303.

61 Cavalheiro EA, Olney JW. Glutamate antagonists: deadly liaisons with cancer. Proc Natl Acad Sci USA 2001;98:5947-8. 